\title{
Covariance analysis, a new approach for relative quantification competitive PCR in evaluation of rumen anaerobic fungal Populations
}

\author{
Mohammad Hadi Sekhavati ${ }^{1}$, Mahdi Elahi Torshizi ${ }^{2, *}$, Mahyar Heydarpour ${ }^{3}$, Adham Fani Maleki ${ }^{4}$ \\ ${ }^{1}$ Department of Animal Science, Ferdowsi University of Mashhad, Iran \\ ${ }^{2}$ Department of Animal Science, Mashhad Branch, Islamic Azad University, Mashhad, Iran \\ ${ }^{3} \mathrm{CABG}$ genomics group, Brigham \& Women's Hospital, Harvard Medical School, USA \\ ${ }^{4}$ Embryonic and Stem Cell Biology and Biotechnology Research Group, Institute of Biotechnology, Ferdowsi University of Mashhad, Iran
}

\section{Email address:}

Sekhavati@um.ac.ir (M. H. Sekhavati), elahi222@mshdiau.ac.ir (M. E. Torshizi), mheydarpour@zeus.bwh.harvard.edu (M. Heydarpour), fanimaleki@gmail.com (A. F. Maleki)

\section{To cite this article:}

Mohammad Hadi Sekhavati, Mahdi Elahi Torshizi, Mahyar Heydarpour, Adham Fani Maleki. Covariance Analysis, a New Approach for Relative Quantification Competitive PCR in Evaluation of Rumen Anaerobic Fungal Populations. Advances in Bioscience and Bioengineering. Vol. 2, No. 5, 2014, pp. 44-50. doi: 10.11648/j.abb.20140205.11

\begin{abstract}
Quantitative competitive polymerase chain reaction (QC-PCR) technique is playing an important role in nucleic acid quantification. This paper describes a new statistical approach for data analyzing in relative quantitative competitive PCR assays. In order to test the accuracy of this statistical model for quantifying anaerobic rumen fungi, samples of rumen fluid were collected from six fistulated Holstein steers which were fed in two different diets groups (soybean meal diet and canola meal diet). Competitor intensity signal (CIS) and efficiency of PCR (EFF) were assumed as two covariates in ANCOVA method. The assumptions for using of these two covariates were tested. A high positive correlation between the mean of the template intensity signal (TIS) through serial dilutions showed an appropriate efficiency of the competitive PCR assays. Results showed that the accuracy of data analyzing for relative quantification anaerobic fungi was considerable improved in ANCOVA model in comparison with ANOVA method and also the power of test is much greater. So, it seems that considering of the CIS and EFF as two co-variables was suitable.
\end{abstract}

Keywords: Analysis of Covariance (ANCOVA), Competitor Intensity Signal (CIS), Efficiency of PCR (EFF), Template Intensity Signal (TIS)

\section{Introduction}

Real-time polymerase chain reaction (RT-PCR) method recently introduced for the rapid quantification of the target DNA sequence[1], however, quantitative competitive PCR(QC-PCR) technique continue to play an important role in nucleic acid quantification because of their significant lower costs of equipment and consumables[2]. QC-PCR is a powerful tool for accurate quantification of DNA or RNA. The procedure relies on the co-amplification of sequence of interest with a serially diluted synthetic DNA fragment of known concentration (competitor) using a single set primers $[3,4]$. The initial quantity of target molecules in the sample can be calculated from the ration of competitor and target derived amplicons generated during PCR, provided that the target and competitor sequences are amplified with equivalent efficiency [5]. The competitor contains the same primer binding site as the target, and the two DNAs compete for reactions to produce PCR products of different size, which can be separated in an agarose gel. The log ratio of intensities of amplified target DNA to competitor is determined by the equation $\log \left(\mathrm{N}_{\mathrm{n} 1} / \mathrm{N}_{\mathrm{n} 2}\right)=\log \left(\mathrm{N}_{01} / \mathrm{N}_{02}\right)+\mathrm{n} \log \left(\mathrm{EFF}_{1} / \mathrm{EFF}_{2}\right)$ [6]. If the efficiencies of amplification $\left(\mathrm{EFF}_{1}\right.$ and $\left.\mathrm{EFF}_{2}\right)$ are equal, the ratio of amplified products $\left(\mathrm{N}_{\mathrm{n} 1} / \mathrm{N}_{\mathrm{n} 2}\right)$ is dependent on the $\log$ ratio of starting products $\left(\mathrm{N}_{01} / \mathrm{N}_{02}\right)$ [6]. The quality of target DNA or cDNA can be most continently assessed at the so-called equivalence point (EQP), at which the target and competitor-derived amplification products display the same signal intensity, indicating identical amounts of target and competitor at the beginning of the PCR reaction [2]. Log ratio of intensities of PCR products is compared to standard curves derived from serial dilutions of known target DNA amplified 
with known amount of competitors [7]. using from this $\operatorname{ratio}\left(\mathrm{Z}=\log \left(\mathrm{N}_{\mathrm{n} 1} / \mathrm{N}_{\mathrm{n} 2}\right)\right)$ is not suitable for statistical analysis in ANOVA method, because template intensity signal (TIS) and competitor intensity signal (CIS) are random variables and then using of this ratio provide assumptive amount of $\mathrm{Z}$ in result. Variation of TIS influence on $Z$ linearly but variation of CIS effect on $\mathrm{Z}$ in hyperbolic way ( $\mathrm{Z}=1 / \mathrm{CIS})$. Variation below the CIS mean has a large effect on $\mathrm{Z}$ than variation above the CIS mean.

Moreover, the magnitude of the error of $\mathrm{Z}$ depends on the error of CIS but also on the absolute value of CIS, in the other way error is higher for low values of CIS. This clearly affects to the homogeneity of variance. The correct way to analyze these ratios is an analysis of covariance (ANCOVA) for the numerator using the denominator of the ratio as covariate. The analysis of covariance (ANCOVA) is a method of adjusting for the effect of an uncontrollable nuisance variable (covariates). The procedure is a combination of analysis of variance and regression analysis. The analysis of covariance involves adjusting the observed response variable for the effect of the covariate variable. Such an adjustment is not performed, the covariate variable could inflate the error mean square and decrease the precision of an experiment $[8,9]$.

Small differences in amplification efficiency (EFF) can have a significant impact on the productivity of PCR. For example, a 5\% difference in amplification efficiency between two targets with identical starting concentration can lead to a twofold difference in amplicon concentration after just 26 cycles [1]. Differences in amplification efficiency between the target and competitor will lead to mistake in relative quantification unless corrections are made and ANCOVA can adjust these differences [1]. An index for evaluation of preference of the new model is obtaining by power of test. The technical definition of power is that it is the probability of detecting a 'true' when it exists. Such tests are useful in determining the number of experimental replicates required to detect a desired numerical difference between, or among, treatments prior to initiating an experiment and also accuracy of an analysis [10].

This paper describes a new model (ANCOVA) for data analysis of quantification competitive PCR for comparing changes of rumen anaerobic fungal population under different treatment. CIS and EFF are two covariates in this study which effect on target intensity band. We evaluated the assumptions of ANCOVA in this analysis and compared ANCOVA model with ANOVA through power of test for each model.

\section{Material and Methods}

\subsection{Isolation and Culturing of Rumen Anaerobic Fungi}

Rumen fungi were isolated from the wheat straw which incubated in fistulae steer. Method of Joblin et al. (1981) was used to grow fungi under anaerobic conditions at $39^{\circ} \mathrm{C}$ for 3 days.

\subsection{Animals, Experimental Design and Diets}

Cows were fed diets that were isoenergetic containing soybean meal (SBM, $n=3)$, canola meal $(C M, n=3)$ from day 5 to 56 postpartum. Six Holstein cows, $560 \pm 4 \mathrm{~kg}$ live weight (Mean \pm SEM) were blocked in pairs based on their previous $305-\mathrm{d}$ milk, parity $\left(2^{\text {nd }}\right.$ and $3^{\text {rd }}$ to $\left.5^{\text {th }}\right)$ and expected calving dates. Six Rumen samples $(200 \mathrm{ml})$ were taken 50 days after feeding each diet, 6 hours after morning feeding. Digesta samples were directly taken from the central portion of the rumen for each cow with initial course filtration through an insect screen with a medium mesh size $(2 \times 1.5 \mathrm{~mm})$. The pooled filtrates, contained digest plant particles and rumen fluid was then stored at $-80^{\circ} \mathrm{C}$ until DNA was extracted for microbial population analysis.

\subsection{DNA Source, PCR Amplification and Construction of Competitor}

Total genomic DNA was isolated from pure culture and rumen fluid samples using Guanidine Thiocyanate-Silica Gel method [11]. The general anaerobic fungal primers (GAF) previously designed by Denman and McSweeney (2006) from multiple alignments of fungal $18 \mathrm{~S}$ ribosomal and ITS1 gene sequences were used in this study and are listed in (Table 1). PCR amplification of rumen anaerobic fungi DNA produces a 110-bp product when amplified with the universal primers. The non-homologous competitor (are listed in Table 1) was designed as described by Sekhavati et al. (2009) which produced a competitor with $191 \mathrm{bp}$ size in PCR reaction.

Table 1. PCR primers for amplifying target and non-homologous competitors.

\begin{tabular}{ll}
\hline Target species & \\
\hline Anaerobic rumen fungi & \\
$\mathrm{GAF}_{1}$ & 5'-GAGGAAGTAAAAGTCGTAACAAGGTTTC-3' \\
$\mathrm{GAF}_{2}$ & 5'-CAAATTCACAAAGGGTAGGATGATTT-5' \\
Entrobacteria phage lambda & \\
LaGAF $_{1}$ & 5'-GAGGAAGTAAAAGTCGTAACAAGGTTTC ${ }^{*}$ GAAGTTCGCAGAATCGTATGTG-3' \\
LaGAF $_{2}$ & 5'-CAAATTCACAAAGGGTAGGATGATTT'GCTGTGGACATAGTTAATCCG-3 \\
\hline
\end{tabular}

The 5' ends of hybrid primers contained a GAF-universal sequence

The PCR mixture contained 50 ng of template DNA, $2 \mu \mathrm{l}$ 10-X PCR buffer, $2.5 \mathrm{mM} \mathrm{MgCl} 2,200 \mu \mathrm{M}$ each dNTPs, 10 pM of each primer and $1 \mathrm{U}$ Taq DNA polymerase. The PCR was performed in a final volume of $25 \mu \mathrm{l}$ sealed in a capillary tip, and thermo cycling was carried out in a model 2000 (Biometra). The PCR amplification condition was as follows: denaturizing at $94^{\circ} \mathrm{C}$ for $4 \mathrm{~min}$ followed by 40 cycles of $94^{\circ} \mathrm{C}$ for $30 \mathrm{~s}, 56^{\circ} \mathrm{C}$ for $30 \mathrm{~s}$, and $72^{\circ} \mathrm{C}$ for $1 \mathrm{~min}$. The PCR products 
were analyzed by running on $2 \%$ agarose gels containing ethidium bromide, and visualized for a single specific band and the absence of primer dimmer products by UV transilluminatin.

\subsection{Quantification of PCR product}

The PCR products were quantified by photographing agarose gels with Polariod 665 film (Polaroid, St. Albans, England), which produce a negative image of the photograph. The negative was scanned with a GS-670 image densitometer (Bio-Rad, Hercules, California) and analyzed with ImageJ $1.38 \mathrm{x}$ software (National Institutes of Health, USA). To correct for differences in the fluorescence of ethidium bromide-stained PCR fragments, the intensities of amplified standard control was multiplied by the ratio 110/191[12].

\subsection{Statistical Analysis and Results}

Data were analyzed with completely randomize design by covariance analysis through Generalized Linear Model (GLM) in SAS program [13].

Statistical model was:

$$
Y_{i j k}=\mu+T_{i}+b_{1}\left(C I S_{i j}-\overline{C I S}\right)+b_{2}\left(e f f_{i j}-\overline{e f f}\right)+e_{i j}+\varepsilon_{i j k}
$$

Where

$\mathrm{Y}_{\mathrm{ij}}=$ template intensity signal

$\mu=$ mean

$\mathrm{T}_{\mathrm{i}}=$ effect of treatment $\mathrm{i}$

$\mathrm{b}_{1} \& \mathrm{~b}_{2}=$ regression coefficients

CIS = control intensity signal

$\mathrm{EFF}=\mathrm{PCR}$ efficiency

$\mathrm{e}_{\mathrm{ij}}=$ experimental error of $\mathrm{j}$ that is related to treatment $\mathrm{i}$

$\dot{\varepsilon}_{\mathrm{ijk}}=$ sampling error of repeated $\mathrm{j}$, related to treatment $\mathrm{i}$ and sample $\mathrm{k}$

For computing the PCR efficiency for each of 18 reactions, the competitor was diluted ( 1 to $10^{-7}$-fold) in distilled $\mathrm{H}_{2} \mathrm{O}$ and amplified by PCR as outlined above. A linear regression of $\log$ ratio of intensities of amplified target DNA to competitor against the concentration of competitor DNA was used for calculation of PCR efficiency (EFF) by using the following equation: $\varepsilon=10^{-1 / \text { slope }}$ for each reaction.

In this model the CIS and the EFF were used as covariate factors (see supplement 1 for programs). Test of the assumptions for ANCOVA model (Independence of CIS and PCR efficiency values from treatments), test for heterogeneity of the slopes and test the normality of residuals were performed using SAS (see supplement2).

A simple ANOVA method without considering of covariable factors also was used in order to compare two methods (see supplement 3).Finally, power of test for both methods (ANOVA \& ANCOVA) was calculated through SAS program (see supplement 4).

\section{Results}

\subsection{Test of Linearity for 6 Dilutions of Standard DNA by Orthogonal Polynomials}

PCR amplification using the anaerobic fungal primers (GAF) produced fungal-specific amplicons of the expected size was confirmed and supported previous study [10]. PCR amplification of the enterobacteria phage lambda DNA using overhang primers (LaGAF) produced the expected size (191 bp) and was purified and used as the standard control. The relative amplification efficiencies of target and standard control DNAs was determined as described. For validation of the competitive PCR method, in addition to molecular test that previously confirmed by Sekhavati et al (2009). The orthogonal polynomial (linear, quadratic and cubic) test was done. Six serial dilutions of standard control $\left(1,10^{-1}, 10^{-2}, 10^{-3}\right.$, $10^{-4}$ and $10^{-5}$ ) were chosen for competitive PCR reaction in each replicate. For testing of statistical validation of competitive PCR reaction, total data which obtained from intensities of target signal (ITS) were analyzed for linearity between 6 dilutions of standard DNA. The orthogonal polynomial contrasts for linear and cubic were significant $(\mathrm{P} \geq 0.01)$ (table 2).

Table 2. Test of linearity between intensities of target signal and serial dilution for validation of QC-PCR method.

\begin{tabular}{lllll}
\hline Contrast & DF & Contrast SS & Mean Square & Pr> F \\
\hline Linear & 1 & 95695.3420 & 95695.3420 & $<0.0001$ \\
Quadratic & 1 & 26.7900 & 26.7900 & 0.7762 \\
Qubic & 1 & 3111.4464 & 3111.4464 & 0.0104 \\
\hline
\end{tabular}

Result of ANCOVA, for output model 1 showed that effects of CIS and EFF (The PCR efficiency calculated for each

replicate ranging from 1.53 to 2.7 , with mean of 1.90 are significant $(\mathrm{p}<0.05)$.

Output model 1

\begin{tabular}{lllll}
\hline Source & DF & Sum of Squares & Mean Square & F Value \\
\hline Model & 10 & 80787.7429 & 8078.7743 & 13.64 \\
Error & 151 & 89463.3446 & 592.4725 & \\
Corrected Total & 161 & 170251.0875 & & \\
\hline
\end{tabular}

R-Square Coeff Var Root MSE TIS Mean

$\begin{array}{llll}0.474521 & 60.22553 & 24.34076 & 40.41601\end{array}$ 


\begin{tabular}{llllll}
\hline Source & DF & Type III SS & Mean Square & F Value & Pr $>$ F \\
\hline Treatment & 2 & 8392.45765 & 4196.22883 & 7.08 & 0.0011 \\
Cow(Treatment) & 6 & 27608.58931 & 4601.43155 & 7.77 & $<.0001$ \\
CIS & 1 & 54893.55099 & 54893.55099 & 92.65 & $<.0001$ \\
EFF & 1 & 2715.29691 & 2715.29691 & 4.58 & 0.0339 \\
\hline
\end{tabular}

Tests of Hypotheses Using the Type III MS for Cow (Treatment) as an Error Term

\begin{tabular}{llllll}
\hline Source & DF & Type III SS & Mean Square & F Value & Pr $>$ F \\
\hline Treatment & 2 & 8392.457653 & 4196.228826 & $\mathbf{0 . 9 1}$ & 0.4510 \\
\hline
\end{tabular}

Assumptions which are necessary for the use of covariance are as follows [13]:

1- Independence of CIS and PCR efficiency values from treatments
2- Test for heterogeneity of the slopes

3- The normality of residuals

\subsection{Independence of CIS and PCR Efficiency Values from Treatments}

Output model 2

\begin{tabular}{llllll}
\hline Source & DF & Type III SS & Mean Square & F Value & Pr $>$ F \\
\hline Treatment & 2 & 2959.2613 & 1479.6306 & 1.03 & 0.3597 \\
\hline
\end{tabular}

Results showed (output model 1 and 2) that the p-value for CIS and EFF are 0.359 and 0.451 respectively and it's clear that co-variables and treatments are independent from each other's. The same result is achieved for co-variable EFF (not show here). So, this assumption was met in our analysis.

\subsection{Test for Heterogeneity of the Slopes}

Output model 3

\begin{tabular}{|c|c|c|c|c|c|}
\hline Source & DF & Sum of Squares & Mean Square & F Value & $\operatorname{Pr}>\mathbf{F}$ \\
\hline Model & 5 & 50326.5667 & 10065.3133 & 13.09 & $<.0001$ \\
\hline Error & 156 & 119924.5208 & 768.7469 & & \\
\hline Corrected Total & 161 & 170251.0875 & & & \\
\hline Source & DF & Type I SS & Mean Square & F Value & $\operatorname{Pr}>\mathbf{F}$ \\
\hline Treatment & 2 & 9672.59900 & 4836.29950 & 6.29 & 0.0024 \\
\hline CIS & 1 & 39979.80143 & 39979.80143 & 52.01 & $<.0001$ \\
\hline CIS*Treatment & 2 & 674.16625 & 337.08313 & 0.44 & 0.6458 \\
\hline Source & DF & Type III SS & Mean Square & F Value & $\operatorname{Pr}>\mathbf{F}$ \\
\hline Treatment & 2 & 3437.89776 & 1718.94888 & 2.24 & 0.1103 \\
\hline CIS & 1 & 39597.80422 & 39597.80422 & 51.51 & $<.0001$ \\
\hline CIS*Treatment & 2 & 674.16625 & 337.08313 & 0.44 & 0.6458 \\
\hline
\end{tabular}

Output model 4

\begin{tabular}{|c|c|c|c|c|c|}
\hline Source & DF & Sum of Squares & Mean Square & F Value & $\operatorname{Pr}>\mathbf{F}$ \\
\hline Model & 5 & 13770.3465 & 2754.0693 & 2.75 & 0.0209 \\
\hline Error & 156 & 156480.7410 & 1003.0817 & & \\
\hline Corrected Total & 161 & 170251.0875 & & & \\
\hline Source & DF & Type I SS & Mean Square & F Value & Pr $>$ F \\
\hline Treatment & 2 & 9672.598999 & 4836.299499 & 4.82 & 0.0093 \\
\hline EFF*Treatment & 2 & 3566.133259 & 1783.066629 & 1.78 & 0.1725 \\
\hline Source & DF & Type III SS & Mean Square & F Value & $\operatorname{Pr}>\mathbf{F}$ \\
\hline Treatment & 2 & 7474.246610 & 3737.123305 & 3.73 & 0.0263 \\
\hline $\mathrm{EFF}$ & 1 & 1666.967396 & 1666.967396 & 1.66 & 0.1993 \\
\hline EFF*Treatment & 2 & 3566.133259 & 1783.066629 & 1.78 & 0.1725 \\
\hline
\end{tabular}

$\mathrm{P}$ value of interaction effect for CIS*treatment and

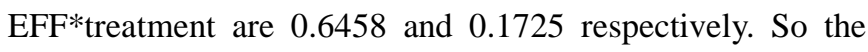
interaction effect of CIS*treatment and $\mathrm{EFF}^{*}$ treatment were not significant, and consequently the hypothesis of homogeneity of slopes was accepted. These interaction effects should be non- significant if the homogeneity of regression assumption is met. In other words, If $\mathrm{p}>0.05$ we do not reject the hypothesis of homogeneity of slopes. 


\subsection{The Normality of Residual}

Output model 5

\begin{tabular}{lll}
\hline Test & statistic & p value \\
\hline Shapiro-Wilk & W 0.94553 & $\operatorname{Pr}<\mathrm{W}<0.0001$ \\
Kolmogorov-Smirnov & D 0.116754 & $\operatorname{Pr}>\mathrm{D}<0.0100$ \\
Cramer-von Mises & W-Sq 0.542727 & $\mathrm{Pr}>\mathrm{W}-\mathrm{Sq}<0.0050$ \\
Anderson-Darling & A-Sq 3.066396 & $\mathrm{Pr}>\mathrm{A}-\mathrm{Sq}<0.0050$ \\
\hline
\end{tabular}

Based on results it is clear that normality index of error that calculates with Shapiro-Wilk test (W) is 0.945 . This number and residual plot show that the error has no specific trend and is completely scattering (Fig1).

Result showed that if we analyze this design with ANOVA method, the power of test is 0.58 but when we include co-variable factors in the model, then the power will increase to 0.73 (see output model 7). Consequently, we can conclude that ANCOVA is better than ANOVA method for relative quantification competitive PCR.

Output model 6

\begin{tabular}{llllll}
\hline Source & DF & Sum of Squares & Mean Square & F Value & Pr $>$ F \\
\hline Model & 8 & 25101.8773 & 3137.7347 & 3.31 & 0.0016 \\
Error & 153 & 145149.2102 & 948.6876 & & \\
Corrected Total & 161 & 170251.0875 & & & \\
\hline
\end{tabular}

R-Square Coeff Var Root MSE TIS Mean

$\begin{array}{llll}0.147440 & 76.20933 \quad 30.80077 \quad 40.41601\end{array}$

Tests of Hypotheses Using the Type III MS for Cow (Treatment) as an Error Term

\begin{tabular}{llllll}
\hline Source & DF & Type III SS & Mean Square & F Value & Pr > F \\
\hline Treatment & 2 & 9672.598999 & 4836.299499 & 1.88 & 0.2322 \\
\hline
\end{tabular}

Output model 7

\begin{tabular}{|c|c|c|c|c|c|c|c|c|c|c|}
\hline Obs & alpha & $\mathbf{a}$ & $\mathbf{n}$ & df1 & df2 & SStrt & MSres & lambda & Fcrit & Power \\
\hline 1 & 0.05 & 3 & 9 & 2 & 6 & 8392.46 & 592 & 14.1764 & 5.14325 & 0.73429 \\
\hline
\end{tabular}

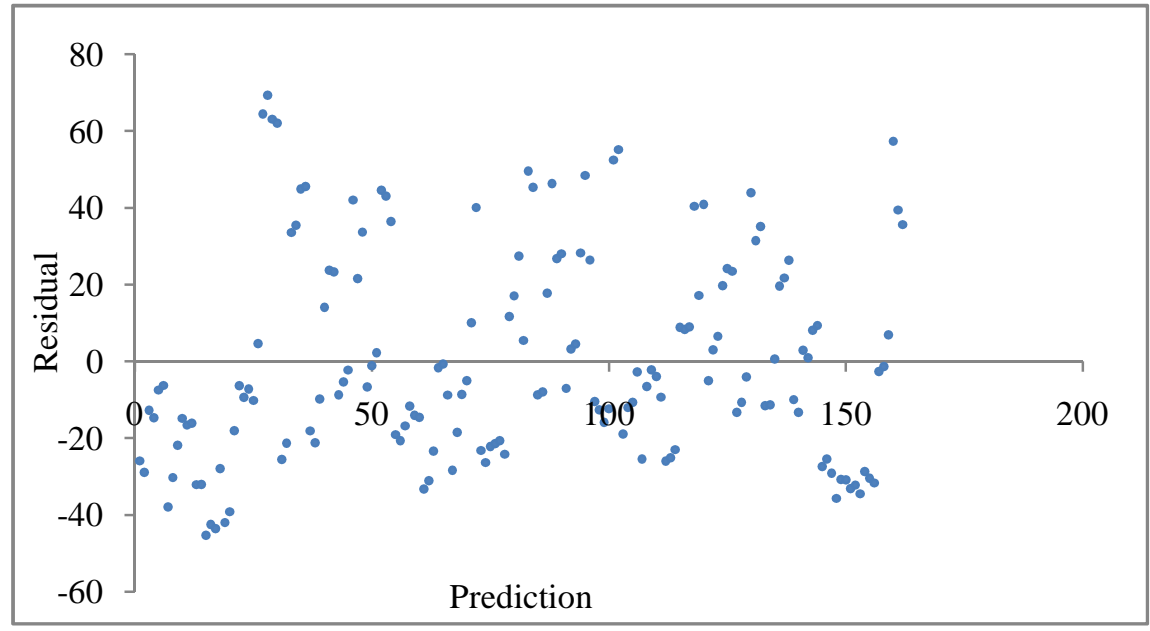

Fig. 1. Residual plot of observations.

\section{Discussion}

Real-time PCR technique as a method for nucleic acid quantification has been widespread in most laboratories worldwide. However, this method still has some limitations in low budget laboratories and technical sophistication [9]. An ideal method that could overcome almost all these limitations is quantitative competitive PCR. This method has a high sensitivity and accuracy in nucleic acid quantification and almost twofold change in target concentration can be detectable by this method [9]. The output data of QC- PCR is the log ratio of intensity of template band to compotator which obtained from image analyzer. These data are not appropriate for statistical analysis with ANOVA model [14]. In this study we tried to introduce a new and simple model for statistical analysis of obtained data which is obtained from QC-PCR. In this regard we conducted a simple experimental design that evaluated an effect of two treatments on rumen anaerobic fungi. In statistical analyses we assumed two factors (CIS and EFF) as co-variables in the model. Results showed a significant effect of these two factors (Output model 1), but we must test the ANCOVA assumption before considering CIS and EFF as co-variables in our model. The results indicated that we could consider these two factors as co-variables (Output 2, 3, 4 and 6). In addition, we validated the QC-PCR method with polynomial contrast vs. the validation of this method that had been done by Sekhavati et al. (2009). They confirmed this method with plotting the 
QC-PCR method against Chitin's results that obtained from cell wall chitin analyses of rumen anaerobic fungi. High significantly of linearity for the mean of TIS through serial dilutions showed the validation of competitive PCR method (Table 2). In addition, significance of cubic trend indicated that we can eliminate the dilution of 1 and $10^{-5}$ from data analyzing because of no significant of intensities signal with adjacent dilutions. So we can perform competitive PCR reaction with 4 dilutions instead of 6 . Plotting the mean intensities of target signal to each serial dilution (Fig.2) confirmed the results of orthogonal contrast.

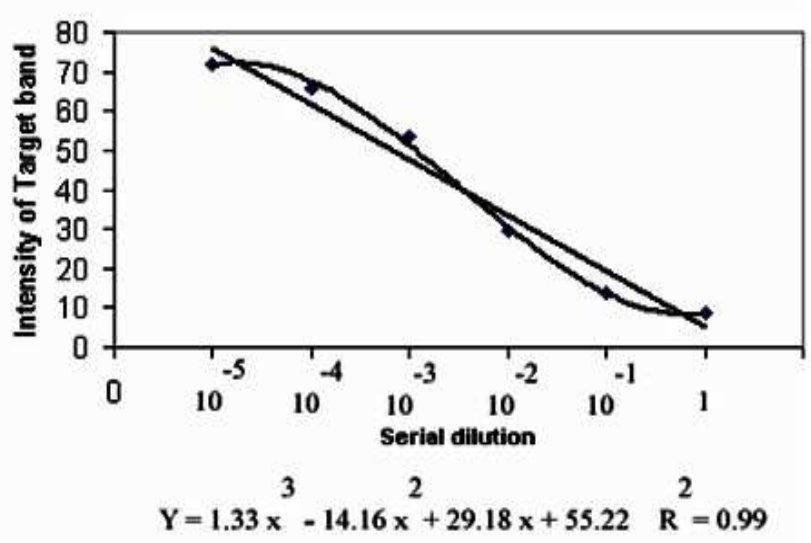

Fig. 2. Linear and cubic manner of mean intensities of target signal to each serial dilution. Each point is consisting 18 PCR reactions for each serial dilution.

In the final step, we compared the power of ANCOVA model with the ANOVA for statistical analysis of rumen anaerobic fungal populations. Results showed that power of test of ANCOVA model is higher than the ANOVA (0.73 vs $0.58)$.

There are lots of studies that have used QC-PCR method for quantifying nucleic acid. However, none of them have used this simple statistical model for analyzing QC-PCR data. In this study we proposed a simple model for data analyzing of QC-PCR data and validation of this model. It seems that ANCOVA model could be a suitable model for data analyzing in QC-PCR method, particularly when the size of data is small.

\section{Acknowledgments}

The authors would like to thank Dr Mohsen Danesh Mesgaran for his help.

\section{Appendices}

\section{Supplement 1:}

DATA EXPERIMET,

INPUT TRETAMENT\$ COW SAMPLE CIS EFF TIS, DATALINES,

DATA,

PROC GLM,
CLASS TREATMENT COW,

MODEL TIS=TREATMENT COW (TREATMENT) CIS EFF,

RANDOM COW (TREATMENT),

TEST H=TREATMENT E=COW (TREATMENT), RUN,

\section{Supplement 2:}

PROC GLM,

TITLE 'TEST FOR INDEPENDENCE OF TREATMENT

AND COVARIABLE',

CLASS TREATMENT,

MODEL CIS OR EFF= TREATMENT,

RUN,

PROC GLM,

TITLE 'TEST FOR HETROGENEITY OF SLOPES',

CLASS TREATMENT,

MODEL TIS $=$ TREATMENT CIS TREATMENT $*$ CIS,

RUN,

PROC GLM,

TITLE 'TEST FOR HETROGENEITY OF SLOPES',

CLASS TREATMENT,

MODEL TIS $=$ TREATMENT EFF TREATMENT $*$ EFF,

RUN,

PROC GLM,

TITLE 'TEST FOR NORMALITY OF RESUALS',

CLASS TREATMENT,

MODEL TIS $=$ TREATMENT CIS EFF,

OUTPUT OUT=CHEK P=PREDI R=RESI,

PROC UNIVARIATE DATA=CHECK NORMAL,

VAR RESI,

PROC PLOT,

PLOT RESI*PREDI=TREATMENT,

RUN,

\section{Supplement 3:}

PROC GLM,

CLASS TREATMENT COW,

MODEL TIS=TREATMENT COW (TREATMENT),

RANDOM COW (TREATMENT),

TEST H=TREATMENT E=COW (TREATMENT), RUN,

\section{Supplement 4:}

DATA POWER,

ALPHA $=0.05$,

$\mathrm{a}=3$,

$\mathrm{n}=9$,

df $1=2$,

df $2=6$,

SSTRT $=8392.457653$,

MSRES $=592$,

LAMBDA $=$ SSTRT/MSRES,

FCRIT $=$ FINV(1-ALPHA,df1,df2),

POWER=1-CDF('F',FCRIT,df1,df2,LAMBDA),

PROC PRINT, RU 


\section{References}

[1] W.M. Freeman, S.J. Walker, and K.E. Vrana, Quantitative RT-PCR: pitfalls and potential. Biotechniques 1999. $26 \mathrm{p}$. 112-125.

[2] F. Watzinger, E. Hörth, and T. Lion, Quantification of $m R N A$ expression by competitive PCR using non-homologous competitors containing a shifted restriction site. Nucleic Acids Research, 2001. 29 p. 52.

[3] A. Gaiger, et al., Increase of bcr-abl chimeric mRNA expression in tumor cells of patients with chronic myeloid leukemia precedes disease progression. Blood 1995. 86 p. 2371.

[4] Joblin, K., Isolation, enumeration, and maintenance of rumen anaerobic fungi in roll tubes. Applied and environmental microbiology, 1981. 42(6): p. 1119-1122.

[5] Vu, H.L., et al., A method for quantification of absolute amounts of nucleic acids by (RT)-PCR and a new mathematical model for data analysis. Nucleic acids research, 2000. 28(7): p. e18-e18.

[6] Zar, J.H., Biostatistical Analysis. Second Edition ed1984: Prentice Hall International, Inc.

[7] Reilly, K. and G. Attwood, Detection of Clostridium proteoclasticumand Closely Related Strains in the Rumen by Competitive PCR. Applied and environmental microbiology, 1998. 64(3): p. 907-913.
[8] G.W, S. and W.G. Cochran, Statistical Methods. Eighth edition ed1991: Iowa state university press/AMES

[9] Zentilin, L. and M. Giacca, Competitive PCR for precise nucleic acid quantification. Nature protocols, 2007. 2(9): p. 2092-2104.

[10] Robinson, P., et al., Some experimental design and statistical criteria for analysis of studies in manuscripts submitted for consideration for publication. Animal feed science and technology, 2006. 129(1): p. 1-11.

[11] Boom, R., et al., Rapid and simple method for purification of nucleic acids. Journal of clinical microbiology, 1990. 28(3): p. 495-503.

[12] Sekhavati, M.H., et al., Development and use of quantitative competitive PCR assays for relative quantifying rumen anaerobic fungal populations in both in vitro and in vivo systems. Mycol Res, 2009. 113(Pt 10): p. 1146-53.

[13] SAS, S. and S.U.s. Guide, Version 9.1. SAS Institute Inc., Cary, NC, 2003.

[14] J. Dubcovsky, http://www.plantsciences.ucdavis.edu/agr205/schedule.htm . Introduction to Analysis of covariance. 